\title{
Carbon Stars in Open Clusters
}

\author{
BERNHARD ARINGER \\ Institut für Astronomie der Universität Wien, Vienna, Austria
}

We have performed a systematic search for carbon stars associated with open clusters. We have taken optical carbon stars from Stephenson's General Catalog of Cool Galactic Carbon Stars and infrared carbon stars from the IRAS LRS Catalog (class $4 \mathrm{n}$ ) and correlated their positions with the open clusters included in the Lyngå catalog. Subsequently all objects that can not be cluster members have been ruled out by investigating their radial velocities, magnitudes, and the probability of an incidental correlation of positions. In the end we came up with several candidates for AGB carbon stars. When it was possible, we calculated absolute bolometric luminosities based on the cluster distances. 